\title{
Entender la otredad desde el saber tradicional
}

\author{
Rogelio Jiménez Marce
}

\author{
Arturo Argueta Villamar, Eduardo \\ Corona M. y Paul Hersch (CoOrds.), 2011 \\ Saberes colectivos y diálogo de saberes en México
}

Centro Regional de Investigaciones Multidisciplinarias-

Universidad Nacional Autónoma de México, Universidad

Iberoamericana-Puebla, Centro Instituto Nacional de

Antropología e Historia-Morelos, México, 574 pp.

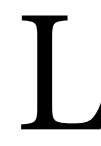

a lectura de Saberes colectivos y diálogo de saberes en México, obra coordinada por Arturo Argueta, Eduardo Corona y Paul Hersch, me recordó un texto que leí hace varios años, en el que dos académicos de la vieja escuela denostaban el saber popular, pues planteaban que el conocimiento producido en las universidades era el único que debía considerarse válido. Ante una posición como la anterior,

\section{Understanding Otherness from Traditional Knowledge}

Rogelio Jiménez Marce: Universidad Iberoamericana-Puebla, Puebla, Puebla, México rojimarc@yahoo.com.mx

Desacatos, núm. 43, septiembre-diciembre 2013, pp. 197-200 debemos congratularnos por la aparición de libros como el que ahora se reseña, pues constituye una evidencia de que es necesario trascender los cerrados círculos científicos para buscar el reconocimiento y la incorporación de todos aquellos conocimientos tradicionales que, sin duda, contribuirán a enriquecer nuestra forma de entender y vivir en el mundo. Pedro Luis Sotolongo y Carlos Jesús Delgado mencionan que el diálogo de saberes busca superar dos situaciones. La primera se refiere al papel hegemónico que la cultura occidental ha mantenido sobre otros saberes y culturas. Un componente indispensable en la superación de las hegemonías cognitivas y 
culturales es el diálogo entre los diferentes grupos y sus saberes. En este sentido, el diálogo implica no sólo aprender del otro sino reconocer que el otro tiene algo que enseñarnos. En lo que respecta a la segunda, se busca trascender los saberes disciplinarios para configurar un saber transdisciplinario, mismo que ayudará a tender puentes conceptuales y metodológicos entre los saberes dialogantes (Sotolongo y Delgado, 2006).

Ahora bien, un primer aspecto que debe destacarse es que este libro forma parte de un esfuerzo colectivo, no sólo medido por el número de autores que participan -34 en 24 artículos-, sino también por el tipo de temas a los que se abocan sus trabajos - las relaciones de las comunidades con las áreas naturales protegidas, la manera en la que se percibe a la flora y a la fauna, la medicina tradicional, los agroecosistemas, la memoria colectiva y sus significados, la educación no formal, entre otros-, así como por el número de instituciones involucradas - la Universidad Nacional Autónoma de México, la Universidad IberoamericanaPuebla, el Instituto Nacional de Antropología e Historia, la Universidad Autónoma del Estado de Morelos, la Benemérita Universidad Autónoma de Puebla y la Universidad Veracruzana-. En este sentido, el libro muestra lo fructífero que puede resultar el trabajo entre diversas universidades y, sobre todo, la colaboración que puede establecerse entre especialistas de diversas ramas del saber. Es evidente que esfuerzos como el que ahora se presenta destacan la urgencia de que la interdisciplinariedad se convierta en un tema de investigación y enseñanza en las agendas universitarias. De hecho, Dimas Floriani advierte que se requiere una nueva revolución científica que conduzca a la destrucción de los sistemas de pensamiento que se han tornado oligopólicos. Para lograr trascender las barreras disciplinarias, según el autor brasileño, es indispensable una reorganización de las universidades, en la que los grupos de investigación sean los que predominen más que los esfuerzos individuales (Floriani, 2011: 105-106).

Ante la diversidad de temas y la riqueza de los aportes de cada uno de los trabajos que conforman el libro, he decidido no hacer una referencia amplia de lo que plantean los autores, tarea imposible en un espacio tan corto como el de una reseña. Más bien me concentraré en tres aspectos que se desprenden de la lectura y que me parecen dignos de ser puntualizados: la cuestión de la interdisciplinariedad, la definición de los conceptos que se utilizan y la pertinencia de incluir un punto de vista histórico en el problema analizado. Sobre el primer asunto, la interdisciplina, quiero mencionar que en 2009 se publicó un número especial de la Revista Mexicana de Sociología en el que la coordinadora, Judit Bokser, enfatizaba que la creciente interacción de las disciplinas había conducido a la revisión de las fronteras del conocimiento y de los paradigmas teóricos que se redefinen, a fin de enfrentar los cambios que se producen en la realidad con recursos conceptuales renovados. $Y$ es que la idea de un solo universo cognitivo ha sido superada y se requiere pensar en la diversidad de universos que afloran, así como en sus interacciones y convergencias. La especialización ha generado, en palabras de Judit Bokser, aislamiento, visiones parroquiales, fronteras inamovibles y cerrazón, factores que han provocado la inhibición de la creatividad y la falta de circulación del conocimiento entre las disciplinas (Bokser, 2009: 52-53, 55-57).

Las ideas anteriores, en mayor o menor medida, son cuestionadas en casi todos los artículos y, como Arturo Argueta lo plantea en la "Introducción" del libro, lo que se busca es tender puentes entre diversas disciplinas con la finalidad de construir un diálogo que no sólo sea interdisciplinario, sino que trascienda hacia lo transdisciplinario, es decir, un diálogo en el que participen en igualdad de circunstancias los académicos, los actores sociales 
y los sectores gubernamentales. Pero lo más importante de todo es que logre establecerse, como lo menciona Víctor Toledo, un verdadero diálogo entre las dos tradiciones del conocimiento humano, diálogo que no debe circunscribirse a un ámbito conceptual o teórico, sino que debe llevarse a espacios concretos y específicos de la realidad. Esta tarea resulta imprescindible para que haya una verdadera comunicación, pues de otra manera sucederá lo que Alberto Betancourt refiere en su artículo: una incapacidad para escuchar, aprender y poner en juego los saberes tanto de una como de otra parte. Si bien es cierto que el conocimiento científico ha tratado de imponerse sobre el tradicional, ahora no debe abogarse por la fórmula contraria, es decir, que lo tradicional se imponga sobre lo científico, sino que más bien debe buscarse que el diálogo de saberes se convierta en una dinámica de saberes en diálogo. Fórmula que, por otra parte, no es inédita ni innovadora pues, como Paul Hersch precisa, las poblaciones en contacto incorporan de manera pragmática elementos de diversa índole, rasgo constitutivo de cualquier proceso civilizatorio que no depende de postulados teóricos, sino de condiciones concretas de vida de las poblaciones.

Sin embargo, no existe un "epistemicidio" como tal, sino que hay diversas maneras de negociar y de apropiarse del conocimiento del otro. Me parece que es pertinente resaltar esta idea, ya que de otro modo no podría explicarse la pervivencia de los saberes tradicionales. Es cierto que existen procesos de subordinación de las ideas, pero no debe pasarse por alto que el conocimiento se transforma de acuerdo con las condiciones históricas y traspasa las barreras socioculturales. Pensemos en la manera en la que se produjo un cambio, no sólo en las representaciones sino también en las prácticas y recursos materiales, cuando los indígenas tuvieron que afrontar la aparición de las enfermedades traídas por los españoles. $\mathrm{O}$, por el contrario, en lo que significó que las mujeres de la alta sociedad española incorporaran los saberes de las indígenas para curar enfermedades o que tuvieran que recurrir a ellas para emplear ciertas prácticas mágicoreligiosas, mismas que, si bien eran perseguidas, no por ello dejaban de ser utilizadas por diversos grupos sociales.

Sobre el segundo punto, la definición de conceptos, me parece que es importante precisar lo que se concibe como saberes colectivos. Arturo Argueta reconoce que en el libro aparecen como sinónimos: "saberes indígenas", "saberes campesinos", "conocimientos tradicionales", "saberes locales" y "sistema de saberes", a los que deben sumarse los términos "sistemas epistémicos indígenas", "saberes colectivos", "saberes tradicionales", "conocimientos locales", "saberes cotidianos", "saberes agrícolas tradicionales", "saberes rurales comunitarios" y "sistemas de conocimiento". La miríada de conceptos, a decir de Argueta, tiene el objetivo de evitar que exista una caracterización homogeneizante, práctica común en la ciencia tradicional. En este mismo sentido, Enrique Leff arguye que la epistemología moderna tiene la pretensión de lograr la "unidad" del conocimiento para llegar a una comprensión general de los fenómenos. Imponer la unicidad en el pensamiento contribuye a crear un pensamiento lineal, único, dominante y hegemónico, el cual tiende a desvalorizar la diversidad, desconocer la diferencia y a no enfrentar la otredad. Sin embargo, como el mismo Leff admite, el diálogo interdisciplinario no puede producirse si no se definen principios conceptuales que hagan posible la articulación de conocimientos comunes, con lo que el biólogo, el médico, el antropólogo, el historiador, el economista, el etnólogo, el ecólogo, el sociólogo y todos los logos restantes tendrán una base epistémica común desde la cual debatir y contribuir al entendimiento de las diversas realidades que se presentan. En 
otras palabras, lo que se debe tratar de retomar es ese saber de fondo que, según Habermas, permite un entendimiento común que dirime las diferencias de intereses, tarea de suma trascendencia, pues como se puede advertir en el libro las maneras de definir los saberes colectivos conllevan al establecimiento de diálogos diferenciados entre los mismos especialistas.

Respecto del tercer punto, la pertinencia de incluir un punto de vista histórico, debo señalar que la perspectiva histórica brilla por su ausencia en el diálogo interdisciplinar que se plantea en el texto. Si bien es cierto que se hacen alusiones al pasado en los artículos de varios de los autores, deben realizarse investigaciones que muestren de qué manera los pueblos modificaron sus conocimientos tras el contacto con la Europa occidental. No cabe duda de que las crónicas y las relaciones indígenas tienen un sesgo, como todo texto producido por una persona que se ubica desde un determinado lugar de producción, según lo define Michel de Certeau (1999), pero la información permitirá reflexionar sobre las representaciones y prácticas de los saberes tradicionales, su difusión entre los nuevos sectores sociales, la represión a la que fueron sometidos, si es que lo fueron, y la manera en la que se transformaron de acuerdo con las nuevas circunstancias sociales, políticas, económicas, culturales e ideológicas. El conocimiento histórico, sin duda, será de utilidad en la cimentación de un "saber de fondo" para profundizar en el diálogo entre las representaciones y prácticas del pasado con las del presente, lo cual ayudará a situar el desarrollo de los saberes colectivos en una perspectiva de larga duración.

Para finalizar, quiero puntualizar que, por la riqueza de las investigaciones, la miríada de perspectivas que incorpora, las herramientas metodológicas que se utilizan y el esfuerzo de unir a instituciones y especialistas de diversas disciplinas, no me cabe la menor duda de que este libro no sólo se convertirá en una referencia obligada para los especialistas de muy variadas áreas del conocimiento, tanto sociales como naturales, sino que también contribuirá a delinear la manera en la que se deben construir los esfuerzos interdisciplinarios, a fin de conseguir equipos de investigación sólidos académicamente, coherentes con su realidad y comprometidos en la búsqueda de un mundo incluyente e inclusivo.

\section{BIBLIOGRAFÍA}

Bokser, Judit, 2009, "Fronteras y convergencias disciplinarias", en Revista Mexicana de Sociología, núm. 71.

De Certeau, Michel, 1999, La escritura de la historia, Universidad Iberoamericana, México.

Floriani, Dimas, 2011, "Interdisciplinaridade e ambiente na América Latina”, en Leila da Costa Ferreira (coord.), A questao ambiental na America Latina. Teoría social e interdisciplinaridade, Editora de Universidade Federal de Campinas, Campinas.

Sotolongo, Pedro Luis y Carlos Jesús Delgado, 2006, La revolución contemporánea del saber $y$ la complejidad social. Hacia unas ciencias sociales de nuevo tipo, Consejo Latinoamericano de Ciencias Sociales, Buenos Aires. 